\title{
Wavelength Conversion Using Polarization Rotation in a Bulk Semiconductor Optical Amplifier
}

\author{
Brendan F Kennedy,, Frederic Surre,, A.L. Bradleyt, Liam Barry,,and Pascal Landais,, \\ 1.School of Electronic Engineering, Dublin City University, Glasnevin, Dublin 9, Ireland \\ 2.Physics Department, Trinity College Dublin, Dublin 2, Ireland. \\ Email address: kennedvb@eeng.dcu.ie
}

As the bandwidth required for optical networks continues to grow all-optical signal processing becomes essential for future optical communications systems. Various techniques have been studied to provide all-optical switching using semiconductor optical amplifiers (SOA s). One such technique is nonlinear polarization rotation (NPR) [1]. This technique results in a rotation of the state of polarization of a linearly polarized signal injected into the device. The effect is caused by birefringence in the device, different losses for orthogonal modes and also an asymmetry in the gain along orthogonal axes, in the case where the active region of the device is subjected to strain.

The aim of the current work is to perform wavelength conversion using the NPR effect [2] and to develop a more detailed understanding of this technique so that the optimum system can be developed. The experiment is a pumpprobe setup where the data carried on the pump is transferred to the continuous-wave $(\mathrm{CW})$ probe by means of the NPR effect. Initially this experiment is performed with a series of pulses representing the optimum data sequence in terms of power. With NPR it is possible to achieve both inverted and non-inverted wavelength conversion. The principle of operation in both cases is that a high intensity pump signal alters the polarization of the probe at the output of the SOA. After the SOA there is a polarization discriminator, consisting of a polarization controller (PC) and a polarizing beam splitter (PBS) aligned in order to transmit, in the case of non-inverted conversion, the probe at the output of the system in the presence of the pump.

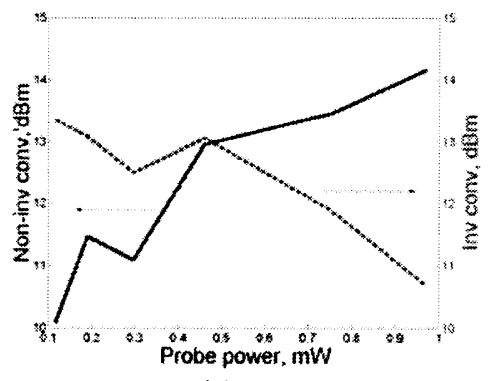

(a)

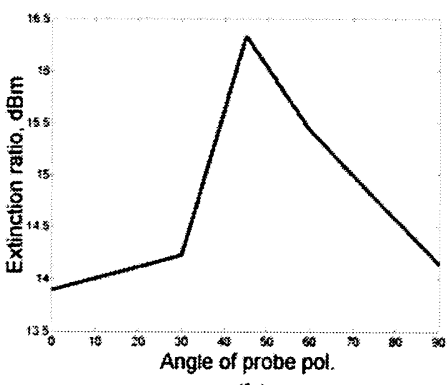

(b)

Fig. 1 Dependence of extinction ratio on (a) probe power (b) probe polarization

The SOA has an active region of length $600 \mu \mathrm{m}$ and a gain peak at $1515 \mathrm{~nm}$. The pulses are generated from a gainswitched DFB laser with external injection and modulated at a frequency of $2.5 \mathrm{GHz}$ with a pulse width of $16 \mathrm{ps}$. The wavelength of the pump laser is $1550.5 \mathrm{~nm}$. The $\mathrm{CW}$ laser is operated at a wavelength of $1538 \mathrm{~nm}$. Both lasers are operated within the $3 \mathrm{~dB}$ gain bandwidth of the SOA.

The maximum achievable extinction ratio (ER) is limited by the ER of the PBS, which is quoted as $20 \mathrm{~dB}$ from the manufacturer. An ER of $18.5 \mathrm{~dB}$ was achieved for the non-inverted conversion and an ER of $20 \mathrm{~dB}$ for inverted conversion. The superior ER for the inverted case is thought to be due to the effect of cross-gain modulation (XGM), which takes place simultaneously with the NPR effect.

From Fig. 1(a) it can be seen that the optimum level of probe power to maximize the ER (y-axis) is dependent on the regime, inverted or non-inverted, of operation. The reason for this result is also due to the XGM effect, which it is desirable to minimize for non-inverted conversion and maximize for inverted conversion. The polarization of the probe has a significant effect on the ER, as can be seen from Fig.1 (b). This result was taken for the inverted setup. A variation in the ER of approx. $2.5 \mathrm{~dB}$ is visible as a function of probe polarization.

[1] B.F. Kennedy, S. Philippe, P. Landais, A.L.Bradley, and H. Soto, Experimental investigation of polarization rotation in semiconductor optical amplifiers , IEE Proc. Optoelectronics, vol.151, no.2, pp.114-118, 2004.

[2] Y. Liu, M.T. Hill, E. Tangdiongga, H. de Waardt, N. Calabretta, G.D. Khoe and H.J.S. Dorren, Wavelength conversion using nonlinear polarization rotation in a single semiconductor optical amplifier , IEEE Phot. Tech. Letters, vol.15, no.1, pp.90-92, 2003. 